Pacific Journal of Mathematic 


\title{
EXTENSIONS OF PRO-AFFINE ALGEBRAIC GROUPS II
}

\author{
Brian Peterson \\ Dedicated to Gerhard Hochschild on the occasion of his 65th birthday
}

Introduction. We fix an algebraically closed field $F$ of characteristic zero throughout. It is known that any proaffine algebraic group $H$ over $F$ is the semidirect product $H_{u} \cdot H_{r}$ of its unipotent radical $H_{u}$ and any maximal reductive subgroup $H_{r}$. This suggests, for considering extensions of a unipotent pro-affine group $U$ over $F$ by $H$, only $H_{u}$ is relevant. More precisely, one is led to ask whether, given a homomorphism $H \rightarrow O(U)=\operatorname{Aut}(U) / \operatorname{Inn}(U)$ for which $\operatorname{Ext}(H, U)$ is nonempty, the restriction map $\operatorname{Ext}(H, U) \rightarrow$ $\operatorname{Ext}\left(H_{u}, U\right)^{H}$ is bijective. The author has shown that this is the case if $U$ is affine. We will show that for unipotent pro-affine $U$, the above restriction map is injective and that it is surjective in the case where $H=H_{u} \times H_{r}$, provided that $\operatorname{Ext}(H, U)$ is nonempty. We will also obtain necessary and sufficient conditions that $\operatorname{Ext}(H, U)$ be nonempty in case both $H$ and $U$ are affine, $U$ unipotent.

The first two results cited above are obtained via the case where $U=A$ is abelian, unipotent and pro-affine (i.e., a pro-vector group). The main obstacle is the fact that the rational cochain groups $C^{n}\left(H_{u}, A\right)$ are not, in general, rational $H_{r}$-modules unless $A$ is affine. This fact necessitates the technical maneuvers of the first three sections.

In $\S 4$, we give a cohomology-free proof that the restriction homomorphism $\operatorname{Ext}(H, A) \rightarrow \operatorname{Ext}\left(H_{u}, A\right)^{H}$ is an isomorphism when all groups are affine, $A$ unipotent and abelian. We also determine when $\operatorname{Ext}(H, U)$ is nonempty, in terms of given homomorphism $H \rightarrow O(U)$, when $H$ and $U$ are affine, $U$ unipotent and not necessarily abelian. The arguments of $\S 4$ where communicated to the author by Gerhard Hochschild, and I am grateful for his allowing me to include them here.

1. Some generalities on inverse limits. Throughout this section, $\mathscr{L}$ is an arbitrary but fixed directed set. All inverse systems and inverse limits have the subscript $\alpha$ ranging over $\mathscr{A} . \mathscr{C}$ is a category whose objects are at least groups and whose morphisms are group homomorphisms, in which $\{0\}$ is the zero object and exactness of a sequence has the usual meaning. The same is true of the category $\mathscr{D}$, which is large enough to contain the image of the inverse limit 
functor lim: $\mathscr{C}^{\prime} \rightarrow \mathscr{D}$ from the category $\mathscr{C}^{\prime}=\mathscr{C}^{\mathscr{N}^{o p}}$ of inverse systems in $\mathscr{C}$ indexed by $\mathscr{A}$. It is well known that $\lim _{\leftarrow}$ is a left exact functor.

We call an object $\left(A_{\alpha}\right)$ of $\mathscr{C}^{\prime}$ compact if it is possible to give each $A_{\alpha}$ a compact $T_{1}$ topology (not necessarily Hausdorff) in such a way that, say, left multiplications are continuous (and hence homeomorphisms) and the maps $A_{\beta} \rightarrow A_{\alpha}$ are continuous and closed when $\alpha<\beta$. Proposition 2.7 of [2] (the projective limit theorem) gives the following lemma.

LEMMA 1.1. Let $0 \rightarrow\left(A_{\alpha}\right) \rightarrow\left(B_{\alpha}\right) \rightarrow\left(C_{\alpha}\right) \rightarrow 0$ be an exact sequence in $\mathscr{C}^{\prime}$ with $\left(A_{\alpha}\right)$ compact. Then the induced sequence $0 \rightarrow A \rightarrow B \rightarrow C \rightarrow 0$ of inverse limits is exact.

Proof. We must show that the map $B \rightarrow C$ is surjective. For this, let $\left(c_{\alpha}\right)$ be an element of $C$. For each $\alpha$, let $X_{\alpha}$ be the set of all $b_{\alpha}$ in $B_{\alpha}$ which are sent to $c_{\alpha}$ by the map $B_{\alpha} \rightarrow C_{\alpha}$. Each $X_{\alpha}$ is nonempty because the maps $B_{\alpha} \rightarrow C_{\alpha}$ are surjective, and the $X_{\alpha}$ 's make an inverse system of sets. Each $X_{\alpha}$ is a coset $\bmod A_{\alpha}$ in $B_{\alpha}$, and we may use any $b_{\alpha}$ in $X_{\alpha}$ to transport the topology of $A_{\alpha}$ to $X_{\alpha}$, the resulting topology on $X_{\alpha}$ being independent of the $b_{\alpha}$ chosen. The maps $X_{\beta} \rightarrow X_{\alpha}$ are continuous and closed when $\alpha<\beta$, so $\lim X_{\alpha}$ is nonempty by the projective limit theorem. Any element $\left.\overleftarrow{\left(b_{\alpha}\right.}\right)$ of $\lim _{\leftarrow} X_{\alpha}$ is sent to $\left(c_{\alpha}\right)$ by the map $B \rightarrow C$.

For the remainder of the section we assume $G$ is a group, $K$ a field, and $\mathscr{C}$ some category of locally finite semisimple $K[G]$-modules which admits all submodules of its objects. We may take $\mathscr{D}$ to be the category of all $K[G]$-modules.

Let $S$ be a system of representatives for the isomorphism classes of simple $K[G]$-modules in $\mathscr{C}$, all of which are finite dimensional. Then each object $M$ of $\mathscr{C}$ is the direct sum $\bigoplus_{s \in S} M^{s}$, where $M^{s}$ is the sum in $M$ of all submodules which are isomorphic with $s$, for $s$ in $S$.

If $\left(M_{\alpha}\right)$ is an object of $\mathscr{C}^{\prime}$ and $M=\lim M_{\alpha}$, we put $M^{[s]}=\lim M_{\alpha}^{s}$ for each $s$ in $S$. Then we have natural $\overleftarrow{\text { maps }} \pi_{s}: M \rightarrow M^{[s]}$ for each $s$ in $S$, and the induced map $M \rightarrow \Pi_{s} M^{[s \mid}$ is easily seen to be injective. We view it as an identification, and then $M$ coincides with the set of all elements $\left(m_{s}\right)=\left(\left(m_{\alpha}^{s}\right)\right)$ of $\Pi_{s} M^{[s]}$ which have, for each $\alpha$, at most finitely many $m_{\alpha}^{s}$ not equal to 0 .

We show next that each $M^{[s]}$ is a (locally finite) semisimple $K[G]$ module all of whose simple submodules are isomorphic with $s$. The kernel $J$ of the representation $\rho: K[G] \rightarrow E(s)=\operatorname{End}_{K}(s)$ has codimension 
at most $\operatorname{dim}(E(s))=\operatorname{dim}(s)^{2}$. Since every element of $s$ is annihilated by $J$, the same is true of every element of $M^{[s]}$. So $M^{[s]}$ is locally finite, and each element of $M^{[s]}$ generates a submodule isomorphic with a quotient of a submodule of $E(s)$, where $E(s)$ has the $K[G]$ module structure for which $g \cdot \phi=\rho(g) \circ \phi$ for $g$ in $G$ and $\phi$ in $E(s)$, which makes $\rho$ a $K[G]$-homomorphism. This corresponds to the action by $K[G]$ on the first factor of $s \otimes s^{*} \cong E(s)$, where $s^{*}=\operatorname{Hom}_{K}(s, K)$. Thus $E(s)$ is a sum of $\operatorname{dim}(s)$ copies of $s$, and $M^{[s]}$ is also a sum of copies of $s$.

If $N$ is a subset of $M$, we put $\bar{N}=M \cap \Pi_{s}\left(\pi_{s}(N)\right)$. Clearly $N \cong \bar{N}$ and $\overline{\bar{N}}=\bar{N}$. We call $\bar{N}$ the closure of $N$ in $M$ and we call $N$ closed in $M$ if $N=\bar{N}$. This does not define a topology on $M$ because (finite) unions of closed subsets need not be closed. However, arbitrary intersections of closed subsets are closed and finite sums of closed submodules (in fact subgroups) are closed.

Lemma 1.2. If $N$ is a closed submodule of $M$, there is a closed submodule $P$ of $M$ with $N+P=M$ and $N \cap P=(0)$.

Proof. Since each $M^{[s]}$ is semisimple, we may choose a $K[G]$ module complement $P^{s}$ to $\pi_{s}(N)$ in $M^{[s]}$. Then $P=M \cap \Pi_{s} P^{s}$ satisfies the requirements of the lemma.

Lemma 1.3. Let $\left(M_{\alpha}\right) \rightarrow\left(N_{\alpha}\right)$ be a $\mathscr{C}^{\prime}$-morphism inducing the map $\pi: M \rightarrow N$ of inverse limits, let $P$ be a closed subset of $N$ and $Q=\pi^{-1}(P)$. Then $Q$ is a closed subset of $M$.

Proof. Let $\left(q^{s}\right)$ be an element of $\bar{Q}$. For each $s$ in $S$, there is a $q$ in $Q$ which projects to $q^{s}$. Now $\pi(q)$ belongs to $P$, hence so does the projection of $\pi(q)$ to $N^{[s]}$ since $P$ is closed. This projection coincides with $\pi\left(q^{s}\right)$, so $q^{s}$ lies in $Q$. Now $\pi\left(\left(q^{s}\right)\right)$ is in $P$, again because $P$ is closed. Thus $\left(q^{s}\right)$ is in $Q$, so $Q=\bar{Q}$.

If $\left(M_{\alpha}\right)$ is an object of $\mathscr{C}^{\prime}$ such that, for each $\alpha$, all but at most finitely many $M_{\alpha}^{s}$ are equal to (0), we say that $\left(M_{\alpha}\right)$ is of finite local type. In this case, if $M=\lim M_{\alpha}$, we have that $M=\Pi_{s} M^{[s]}$.

Lemma 1.4. Let $\left(M_{\alpha}\right) \rightarrow\left(N_{\alpha}\right)$ be a $\mathscr{C}^{\prime}$-morphism and suppose that $\left(M_{\alpha}\right)$ is of finite local type. Then the image of the induced map $\pi: M \rightarrow N$ of inverse limits is a closed submodule of $N$.

Proof. Denote $\pi(M)$ by $P$ and suppose $\left(p^{s}\right)$ is in $\bar{P}$. For each $s$ in $S$, there is a $p$ in $P$ which projects to $p^{s}$. Say $\pi\left(m_{s}\right)=p$ where $m_{s}=\left(m_{s}^{t}\right) \in \Pi_{t \in S} M^{[t]}=M$. Then clearly $\pi\left(m_{s}^{s}\right)=p^{s}$. Since $M=\Pi_{s} M^{[s]}$, 
$\left(m_{s}^{s}\right)$ belongs to $M$. Now $\pi\left(\left(m_{s}^{s}\right)\right)=\left(p^{s}\right)$, so $\left(p^{s}\right)$ is in $P$ and $P=\bar{P}$.

For any $G$-module $A$, let $B^{n}(G, A), Z^{n}(G, A)$, and $H^{n}(G, A)$ be the usual groups of $n$-coboundaries, $n$-cocycles, and $n$-cohomology classes for $G$ in $A$.

LemmA 1.5. Let $\left(M_{\alpha}\right)$ be an object of $\mathscr{C}^{\prime}$ and $M=\lim M_{\alpha}$. Then the natural map $H^{1}(G, M) \rightarrow \lim H^{1}\left(G, M_{\alpha}\right)$ is injective.

Proof. We have a commutative diagram with exact rows

$(0)$

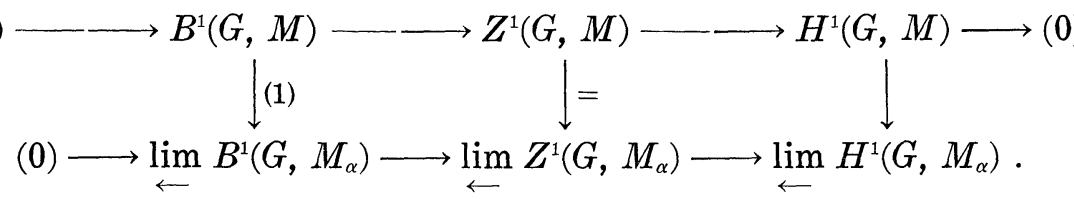

To prove the lemma, we must show that (1) is surjective. For this we consider the commutative diagram with exact rows

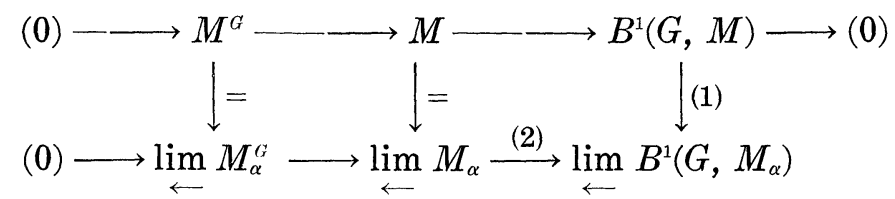

where the superscript $G$ denotes $G$-fixed part. The lemma is equivalent to showing that (2) is surjective. Since each $M_{\alpha}$ is semisimple, we have the usual decompositions $M_{\alpha}=M_{\alpha}^{G} \oplus\left(M_{\alpha}\right)_{G}$ where $\left(M_{\alpha}\right)_{G}$ is the set of sums of elements of the form $g \cdot m-m$ with $g$ in $G$ and $m$ in $M_{\alpha}$. The inverse system $\left(M_{\alpha}\right)$ respects these decompositions, so $M=M^{G} \oplus M_{[G]}$ where $M_{[G]}=\lim \left(M_{\alpha}\right)_{G}$. Since the map $\left(M_{\alpha}\right)_{G} \rightarrow B^{1}\left(G, M_{\alpha}\right)$ is an isomorphism for each $\alpha$, the map $M_{[G]} \rightarrow$ $\lim B^{1}\left(G, M_{\alpha}\right)$ is also an isomorphism, and the lemma is proved.

2. Injectivity of the restriction homomorphism $\operatorname{Ext}(H, A) \rightarrow$ $\operatorname{Ext}\left(H_{u}, A\right)$ for unipotent abelian $A$. Throughout this and the next section, $H$ is a pro-affine algebraic group over $F$ and $A$ is a unipotent abelian pro-affine $H$-group (i.e., $H$ operates on $A$ and the induced map $H \times A \rightarrow A$ is a polynomial map). We write $A=\lim A_{\alpha}$ where the $A_{\alpha}$ 's are the restriction images of $A$ to a cofinal family of finitely generated $H$-stable Hopf subalgebras of the polynomial algebra $P(A)$ of $A$.

Lemma 2.1. The natural map $H^{1}(H, A) \rightarrow \lim H^{1}\left(H, A_{\alpha}\right)$ is an isomorphism. 
Proof. We remined the reader that, as in [1], $H^{1}(H, A)$ and $H^{1}(H, A)$ and $H^{1}\left(H, A_{\alpha}\right)$ are built from polynomial 1-cocycles, though our proof also applies to the 1-cohomology groups built from arbitrary 1-cocycles. We consider two commutative diagrams, both with exact rows,

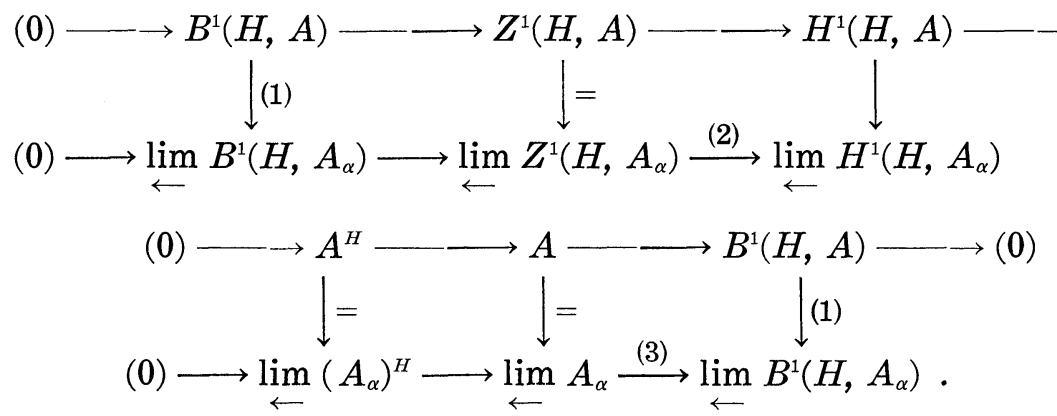

To see that the map of the lemma is surjective, we must show that (2) is surjective. To see that it is injective, we must show that (1) is surjective, or equivalently that (3) is surjective. The surjectivity of both (2) and (3) follows from Lemma 1.1, since each $\left(A_{\alpha}\right)^{H}$ (respectively $\left.B^{1}\left(H, A_{\alpha}\right)=A_{\alpha} /\left(A_{\alpha}\right)^{H}\right)$ is a finite dimensional vector group and the coset topology (in which the closed sets are the finite unions of translates of subspaces) makes the lemma applicable. This completes the proof.

We recall that $H_{u}$ denotes the unipotent radical of $H$.

THEOREM 1. The restriction homomorphism $\operatorname{Ext}(H, A) \rightarrow$ $\operatorname{Ext}\left(H_{u}, A\right)$ is injective.

Proof. From [4] we have an exact sequence $\operatorname{Ext}\left(H / H_{u}, A^{I I} u\right) \rightarrow$ $\operatorname{Ext}_{H_{u}}(H, A) \rightarrow H^{1}\left(H / H_{u}, H^{1}\left(H_{u}, A\right)\right)$ where $H^{1}\left(H_{u}, A\right)$ is built from polynomial 1-cocycles $H_{u} \rightarrow A$ while $H^{1}\left(H / H_{u}, H^{1}\left(H_{u}, A\right)\right)$ is buit from arbitrary 1-cocycles $H / H_{u} \rightarrow H^{1}\left(H_{u}, A\right)$. Since $H / H_{u}$ is reductive and $A^{I I} u$ unipotent, we have that $\operatorname{Ext}\left(H / H_{u}, A^{H_{u}}\right)=(0)$. So to show that the kernel $\operatorname{Ext}_{H_{u}}(H, A)$ of the restriction homomorphism is trivial, we must show that the map $\operatorname{Ext}_{H_{u}}(H, A) \rightarrow H^{1}\left(H / H_{u}, H^{1}\left(H_{u}, A\right)\right)$ is the zero map.

For each $\alpha$, we have a commutative diagram

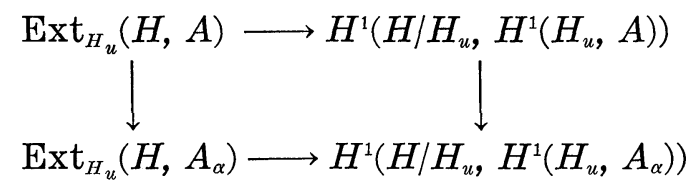

and, since each $A_{\alpha}$ is an affine $H$-group, Proposition 5.2 of [1] shows that each $\operatorname{Ext}_{H_{u}}\left(H, A_{\alpha}\right)=(0)$. Hence the map 


$$
\operatorname{Ext}_{H_{u}}(H, A) \longrightarrow \lim _{\leftarrow} H^{1}\left(H / H_{u}, H^{1}\left(H_{u}, A_{\alpha}\right)\right)
$$

induced by these is the zero map. This latter map is a composite

$$
\begin{aligned}
\operatorname{Ext}_{H_{u}}(H, A) & \longrightarrow H^{1}\left(H / H_{u}, H^{1}\left(H_{u}, A\right)\right) \longrightarrow H^{1}\left(H / H_{u}, \lim _{\leftarrow} H^{1}\left(H_{u}, A_{\alpha}\right)\right) \\
& \longrightarrow \lim _{\leftarrow} H^{1}\left(H / H_{u}, H^{1}\left(H_{u}, A_{\alpha}\right)\right) .
\end{aligned}
$$

The second map is an isomorphism by Lemma 2.1. Since each $H^{1}\left(H_{u}, A_{\alpha}\right)$ is a (locally finite) rational module for the reductive group $H / H_{u}$, and hence semisimple, Lemma 1.5 shows the third map is injective. It follows that the first map is the zero map, and the theorem is proved.

COROLLARY 1. Suppose $H$ and $U$ are pro-affine algebraic groups over $F, U$ unipotent, and a homomorphism $H \rightarrow O(U)=\operatorname{Aut}(U) / \operatorname{Inn}(U)$ is given so that $\operatorname{Ext}\left(H_{u}, U\right)$ is nonempty. Then the restriction map $\operatorname{Ext}(H, U) \rightarrow \operatorname{Ext}\left(H_{u}, U\right)$ is injective.

Proof. Let $A$ denote the center of $U$. Then $\operatorname{Ext}(H, A)$ operates on $\operatorname{Ext}(H, U)$ and $\operatorname{Ext}\left(H_{u}, A\right)$ operates on $\operatorname{Ext}\left(H_{u}, U\right)$, both via the usual Baer composition. In view of the compatability of these actions with respect to the restriction maps given by Proposition 2.4 of [1], the corollary follows immediately from the fact that the first action is transitive and the second faithful. In fact, both actions are faithful and transitive by Proposition 2.1 of [1].

3. A special case of surjectivity of the restriction homomorphism $\operatorname{Ext}(H, A) \rightarrow \operatorname{Ext}\left(H_{u}, A\right)^{H}$. We preserve all notations of $\S 2$. Proposition 5.1 of [1] shows that in the case where $A$ is affine, the image of the restriction homomorphism coincides with the $H$-fixed part $\operatorname{Ext}\left(H_{u}, A\right)^{H}$ of $\operatorname{Ext}\left(H_{u}, A\right)$. The proof proceeds by choosing a maximal reductive subgroup $H_{r}$ of $H$ and then an $H_{r}$-stable complement $S$ for $B^{2}\left(H_{u}, A\right)$ in $Z^{2}\left(H_{u}, A\right)$. This is possible because $Z^{2}\left(H_{u}, A\right)$ is a locally finite rational module for the reductive group $H_{r}$, and hence semisimple. This ceases to be the case, in general, when $A$ is no longer affine. Given an extension of $A$ by $H_{u}$ whose class is $H$-fixed, the proof goes on to select the representative in $S$ for the extension, which is necessarily $H_{r}$-fixed. From this $H_{r}$-fixed 2-cocycle, one can construct an extension of $A$ by $H$ which restricts to the given extension. We therefore have everything that is needed to prove the following theorem.

THEOREM 2. If $A$ is a unipotent abelian pro-affine H-group (over $F$ ) and $H=H_{u} \times H_{r}$ (i.e., $H_{u}$ and $H_{r}$ commute), then the image 
of the restriction homomorphism coincides with $\operatorname{Ext}\left(H_{u}, A\right)^{H}$.

Proof. It suffices to obtain an $H_{r}$-stable complement for $B^{2}(H, A)$ in $Z^{2}\left(H_{u}, A\right)$. We have that $C^{1}\left(H_{u}, A\right)=\lim C^{1}\left(H_{u}, A_{\alpha}\right)$ for the spaces of polynomial 1-cochains, and each $C^{1}\left(H_{u}, A_{\alpha} \overleftarrow{)}=A_{\alpha} \otimes P\left(H_{u}\right)\right.$ is a rational $H_{r}$-module. An element $x$ of $H_{r}$ acts on $f$ in $C^{1}\left(H_{u}, A_{\alpha}\right)$ so that $(x \cdot f)(h)=x \cdot f\left(x^{-1} h x\right)$ for $h$ in $H_{u}$. Since $H_{u}$ and $H_{r}$ commute, the inside action is trivial. Thus $H_{r}$ acts on $A_{\alpha} \otimes P\left(H_{u}\right)$ via the first factor only. Each $A_{\alpha}$ involves only finitely many isomorphism classes of simple $H_{r}$-modules, so the same is true of each $A_{\alpha} \otimes P\left(H_{u}\right)$. Thus the $C^{1}\left(H_{u}, A_{\alpha}\right)$ 's form an inverse system of finite local type in the sense of $\S 1$, and Lemma 1.4 shows that the image $B^{2}\left(H_{u}, A\right)$ of the boundary map $C^{1}\left(H_{u}, A\right) \rightarrow Z^{2}\left(H_{u}, A\right)=\lim Z^{2}\left(H_{u}, A_{\alpha}\right)$ is a closed submodule. Then Lemma 1.2 guarantees $\overleftarrow{t}$ the existence of the desired complement, so the theorem is proved.

CoRollary 2. If, with the notations and assumptions of Corollary 1 , we have $H=H_{u} \times H_{r}$, then the image of the restriction map $\operatorname{Ext}(H, U) \rightarrow \operatorname{Ext}\left(H_{u}, U\right)$ coincides with $\operatorname{Ext}\left(H_{u}, U\right)^{H}$.

Proof. Again we let $A$ denote the center of $U$. If $\xi$ and $\xi^{\prime}$ represent elements of $\operatorname{Ext}\left(H_{u}, A\right)$ and $\operatorname{Ext}\left(H_{u}, U\right)$, respectively, then as was observed in [1], we have $x \cdot\left(\xi+\xi^{\prime}\right)=x \cdot \xi+x \cdot \xi^{\prime}$ for $x$ in $H$, where + indicates the Baer action. The corollary follows immediately from this observation, the theorem, and the transitivity of the Baer action.

4. A sharper treatment in the affine case. Let $H$ and $U$ be affine algebraic groups over $F, U$ unipotent, and $\eta: H \rightarrow O(U)$ a homomorphism. If there does exist an extension $U \rightarrow E \rightarrow H$ inducing $\eta$, the extension is necessarily $H_{r}$-split because $H_{r}$ is reductive and $U$ unipotent. Since $U$ is unipotent and affine, the sequence $\operatorname{Inn}(U) \rightarrow$ $\operatorname{Aut}(U) \rightarrow O(U)$ is a sequence of morphisms of affine algebraic groups over $F$. The $H_{r}$-splitting of the above extension shows, in particular, that the restriction of $\eta$ to $H_{r}$ is a rational homomorphism $H_{r} \rightarrow$ $O(U)$. We will now show that this necessary condition is also sufficient in the following sense.

THEOREM 3. If $H$ and $U$ are affine algebraic groups over $F, U$ unipotent, and the restriction $\eta^{\prime}$ of the homomorphism $\eta: H \rightarrow O(U)$ to a maximal reductive subgroup $H_{r}$ of $H$ is a rational homomorphism $H_{r} \rightarrow O(U)$, then the restriction map $\operatorname{Ext}(H, U) \rightarrow \operatorname{Ext}\left(H_{u}, U\right)^{H}$ is surjective. In particular, $\operatorname{Ext}(H, U)$ is nonempty if $\operatorname{Ext}\left(H_{u}, U\right)^{H}$ 
is nonempty.

Proof. Let $\tau: \operatorname{Aut}(U) \rightarrow O(U)$ be the canonical map and put $R=$ $\tau^{-1}\left(\eta^{\prime}\left(H_{r}\right)\right)$. Since $\eta^{\prime}\left(H_{r}\right)$ is reductive and $\operatorname{Inn}(U)$ unipotent, the extension $\operatorname{Inn}(U) \rightarrow R \rightarrow \eta^{\prime}\left(H_{r}\right)$ is split. So $\eta^{\prime}$ lifts to a rational homomorphism $\mu: H_{r} \rightarrow \operatorname{Aut}(U)$. Let $\sigma: H_{r} \rightarrow \operatorname{Aut}(U) \times \operatorname{Aut}\left(H_{u}\right)$ be induced by $\mu$ and conjugations on $H_{u}$ (within $H$ ).

Now let $\xi:\left[U, E, H_{u}\right]_{\alpha, \pi}$ be an extension inducing the restriction of $\eta$ to $H_{u}$ whose class is $H$-fixed. For each $x$ in $H_{r}$, the extension $x \cdot \xi$ map is defined (as in [1]) as $x \cdot \xi=\left[U, E, H_{u}\right]_{\alpha^{\prime}, \pi^{\prime}}$ where $\alpha^{\prime}=$ $\alpha \circ \mu\left(x^{-1}\right)$ and $\pi^{\prime}(e)=x \pi(e) x^{-1}$ for $e$ in $E$. An equivalence $x \cdot \xi \rightarrow \xi$ is a rational automorphism of $E$ which stabilizes $U$, on which it induces $\mu(x)$, and which induces conjugation by $x$ on $H_{u}$.

The set $\operatorname{Aut}^{0}(E)$ of rational automorphisms of $E$ stabilizing $U$ is an algebraic subgroup of the algebraic group $\operatorname{Aut}(E)$ and we have the natural rational homomorphism $\lambda: \operatorname{Aut}^{0}(E) \rightarrow \operatorname{Aut}(U) \times \operatorname{Aut}\left(H_{u}\right)$. The assumption that the class of $\xi$ is $H$-fixed says precisely that $\sigma\left(H_{r}\right) \cong \lambda\left(\operatorname{Aut}^{0}(E)\right)$. The kernel $P$ of $\lambda$ is unipotent, as is most easily seen via the isomorphism $\operatorname{Aut}(E)=\operatorname{Aut}(L(E))$, where $L(E)$ is the Lie algebra of $E$. Choosing a basis for $L(E)$ containing one for $L(U), P$ operates by unipotent matrices. So we put $S=\lambda^{-1}\left(\sigma\left(H_{r}\right)\right)$ and argue as above that the extension $P \rightarrow S \rightarrow \sigma\left(H_{r}\right)$ splits, so that $\sigma$ lifts to a rational homomorphism $\nu: H_{r} \rightarrow A u t^{0}(E)$. Now if $E \cdot H_{r}$ is the semidirect product with respect to $\nu$, then $U \rightarrow E \cdot H_{r} \rightarrow H_{u} \cdot H_{r}=H$ is an extension inducing $\eta$ which restricts to $\xi$. This completes the proof.

The only serious obstacle to using the same argument in the proaffine case is the fact that $\operatorname{Aut}(E)$ need not be a (pro-affine) algebraic group when $E$ is a unipotent pro-affine group over $F$ (cf. [5]). Suppose that $H, U$, and $\eta$ are as above except that that $H$ and $U$ are proaffine and not necessarily affine. Assume we have the necessary condition that the restriction of $\eta$ to $H_{r}$ is a rational homomorphism onto the image in $O(U)$ of an algebraic subgroup of $\operatorname{Aut}(U)$. Then we obtain $\mu: H_{r} \rightarrow \operatorname{Aut}(U)$ lifting the restriction of $\eta$ to $H_{r}$ just as above. Let us call (the class of) an extension $\xi=\left[U, E, H_{u}\right]$ strongly $H$-fixed if there is an algebraic subgroup of $\operatorname{Aut}^{\circ}(E)$ whose image in $\operatorname{Aut}(U) \times \operatorname{Aut}\left(H_{u}\right)$ contains $\sigma\left(H_{r}\right)$, where $\sigma$ is defined as above. Then, for a strongly $H$-fixed extension, the above argument works with only slight modification, owing to the fact that $P$ need not be an algebraic subgroup of $\operatorname{Aut}(E)$. So the image of the restriction $\operatorname{map} \operatorname{Ext}(H, U) \rightarrow \operatorname{Ext}\left(H_{u}, U\right)$ coincides with the set of strongly $H$ fixed elements of $\operatorname{Ext}\left(H_{u}, U\right)$. We do not know whether every $H$ fixed element of $\operatorname{Ext}\left(H_{u}, U\right)$ is strongly $H$-fixed. By Corollary 2, we 
see that this is the case when $H=H_{u} \times H_{r}$ provided $\operatorname{Ext}(H, U)$ is nonempty, or equivalently provided at least one element of $\operatorname{Ext}\left(H_{u}, U\right)$ is strongly $H$-fixed.

\section{REFERENCES}

1. B. Peterson, Extensions of pro-affine algebraic groups, Pacific J. Math., 77 (1979), 189-231.

2. G. Hochschild and G. D. Mostow, Representations and representative functions of Lie groups, Annals of Math., 66 (1957), 495-542.

3. - Pro-affine algebraic groups, Amer. J. Math., XCI (1969), 1127-1140.

4. G. Hochschild, Basic Constructions in Group Extension theory, Contributions to algebra, dedicated to Ellis Kolchin on the occassion of his 60th birthday, Academic Press, 1977.

5. - Algebraic automorphism groups, Illinois J. Math., 19 (1975), 131-144.

Received February 1, 1978. We also acknowledge support under NSF grant number MCS 77-03939.

San Jose State University

SAN Jose, CA 95192 



\section{PACIFIC JOURNAL OF MATHEMATICS}

\section{EDITORS}

DONALD BABBITT (Managing Editor)

University of Galifornia

Los Angeles, California 90024

HUGo RossI

University of Utah

Salt Lake City, UT 84112

C. C. MOORE AND ANDREW OGG

University of California

Berkeley, CA 94720
J. DUGUNDJI

Department of Mathematics University of Southern California Los Angeles, California 90007

R. FINN AND J. MILGRAM Stanford University Stanford, California 94305

\section{ASSOCIATE EDITORS}

E. F. BECKENBACH

B. H. NEUMANN

F. WOLF

K. YosHIDA

\section{SUPPORTING INSTITUTIONS}

UNIVERSITY OF BRITISH COLUMBIA CALIFORNIA INSTITUTE OF TECHNOLOGY UNIVERSITY OF CALIFORNIA MONTANA STATE UNIVERSITY UNIVERSITY OF NEVADA, RENO NEW MEXICO STATE UNIVERSITY OREGON STATE UNIVERSITY UNIVERSITY OF OREGON
UNIVERSITY OF SOUTHERN CALIFONIA STANFORD UNIVERSITY UNIVERSITY OF HAWAII UNIVERSITY OF TOKYO UNIVERSITY OF UTAH WASHINGTON STATE UNIVERSITY UNIVERSITY OF WASHINGTON 


\section{Pacific Journal of Mathematics}

\section{Vol. 86, No. $1 \quad$ November, 1980}

Gert Einar Torsten Almkvist, Invariants, mostly old ones .............. 1

Hyman Bass, Groups of integral representation type ................ 15

A. Białynicki-Birula, On action of SL(2) on complete algebraic

varieties........................................

Frederick Paul Greenleaf and Martin Allen Moskowitz, Groups of

automorphisms of Lie groups: density properties, bounded orbits, and

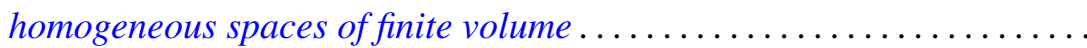

Raymond Taylor Hoobler, A cohomological interpretation of Brauer groups

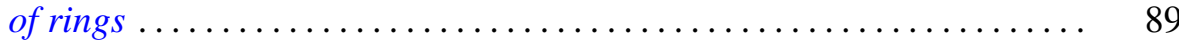

Irving Kaplansky, Superalgebras ........................ 93

Jerrold Lewis Kleinstein and Alex I. Rosenberg, Succinct and

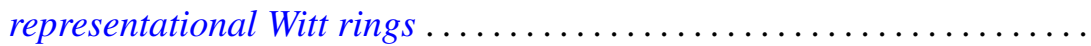

E. R. Kolchin, On universal extensions of differential fields ............ 139

Andy R. Magid, Analytic subgroups of affine algebraic groups. II ....... 145

Calvin Cooper Moore, The Mautner phenomenon for general unitary

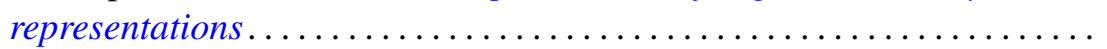

George Daniel Mostow, On a remarkable class of polyhedra in complex hyperbolic space ................................ 171

Brian Lee Peterson, Extensions of pro-affine algebraic groups. II . . . . . . 277

John Henry Reinoehl, Lie algebras and affine algebraic groups......... 287

Maxwell Alexander Rosenlicht, Differential valuations .

John Brendan Sullivan, The second Lie algebra cohomology group and Weyl modules..................................... 321

Moss Eisenberg Sweedler, Right derivations and right differential

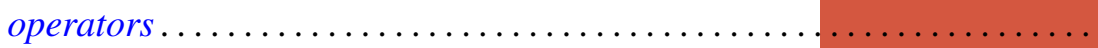

Bostwick Frampton Wyman, Time varying linear discrete-time systems. II. Duality.................................. 